\title{
Digital chemsex publics: \\ Algorithmic and user configurations of fear and desire on Pornhub
}

\author{
Author: Kristian Møller ORCID: https://orcid.org/0000-0003-0056-3471 \\ Accepted for publication in European Journal of Cultural Study \\ https://doi.org/10.1177/13675494211006679
}

Suggested citation: Møller, K. (2021). Digital chemsex publics: Algorithmic and user configurations of fear and desire on Pornhub. European Journal of Cultural Studies. https://doi.org/10.1177/13675494211006679

\begin{abstract}
In recent years, chemsex has emerged as both a subcultural vernacular and an orientation device for gay health promotion. Chemsex loosely describes gay men using certain drugs to extend and modulate group sex practice. In line with hegemonic responses to gay sexuality in general, most research has been grounded in problematisation, with discourse mostly returning to the question of containment. Drawing on porn, platform and critical drug studies, the study offers a corrective approach, this article defines a networked, cultural study of chemsex that is attuned to how chemsex erotics operate in many different (digital) intimate publics. Assembling algorithmic search suggestions, 41 videos and 450 comments, the article finds that the videos and comments found through the search function are vastly different than those found through user-generated playlists. Two competing publics form around the fear/desire-response to drug use: a cautious erotic of disinhibition, and a counterpublic erotic of transgression.
\end{abstract}

Keywords: porn, platform studies, Pornhub, counterpublic, drugs, gay, transgression, disinhibition.

\section{Introduction}

How is this chemsex?? (user comment to the Pornhub video "SLAMMED DADDY AND BOYS CHEMSEX SWEAT FUCK.")

I have arrived at this comment on the Pornhub video service after searching for the term "chemsex", which describes the emerging phenomenon of gay men using the drugs methamphetamine ("crystal meth"), GHB, and/or mephedrone to temporally extend, and affectively intensify, group sex. The commenter is reacting to a 12-minute long video clip, in which three muscular and burly men have intense sex: one man is fucked by the two others taking turns, with them passionately dominating him both physically and verbally. Notably, no drug-taking is ever shown, nor is there any mention of it. Hence, the user comment seems warranted: when no drugs can be seen, and when the onscreen sex looks like "regular" amateur gay porn, how is this chemsex? This raises the notion that you cannot always simply "see" chemsex because the performer might be high in ways that escape 
reliable visual verification. With this realisation in mind, we should instead ask how this video becomes chemsex. Despite the video not depicting acts of drug-taking explicitly, crucially it still emerges from my search for chemsex. Furthermore, because the video's extra-textual elements invite drug use as a decoding strategy for the pleasure of the performers, I suggest that it encourages us to consider how the boundaries of chemsex as a social imaginary needs to be expanded to include a wider range of (digital) subjects, objects, and practices.

In addressing the above, this paper explores how chemsex emerges on the online porn platform Pornhub. In the relatively short time drug use in gay male group sex has been conceptualised as "chemsex", most academic work has addressed the phenomenon from an epidemiological perspective (see Bourne et al., 2014, 2015). This orientation seems warranted by developments in the socio-material realities of mostly urban gay men, who are increasingly organising their sexual life around private gatherings in which new, drugged socio-sexual relations emerge. While this work is key to formulating institutional responses that address these issues, its ultimate focus on "problematic" chemsex runs the risk of normatively containing "problem publics" from "respectable" ones, thereby in turn cutting off flows of care, empathy and even recognition. Literature within cultural critical drug studies have criticised such simplistic, binary framings, taking alternative routes for exploring what the category of chemsex means and how it surfaces. They show chemsex (or chillout, wired play, or Party'n'Play, depending on the national context) to be a space for specific negotiations and explorations of what drugged bodies are able to feel and do. Through a combination of queer theory and ANT, Race (2018) finds that the capacities of crystal meth go hand in hand with screen-based sexual sociability, both in terms of its connective affordances and its requirements to multitask. On a different scale, Hakim (2018) shows how the intimate potentials of chemsex in London emerge as a response to the pressures on gay spaces (rent increases outpricing gay bars) and the pressures of entrepreneurial individualism on gay people's capacity to create and sustain networks of care.

More generally, queer theory has taught us that the very problematisation of gay sexuality is not new, but in fact key to understanding both hegemonic culture and subcultural gay erotic organisation. Sedgwick (1985) has shown how masculinity operates in close proximity to the threat of homosexual transgression, that the homosexual figure is indeed crucial to understanding homosociality. Further, in gay studies, Dean's seminal work on barebacking (Dean, 2009) proposes that "transgressive" gay male sex cultures are shot through with care, and that their affective registers are subculturally produced in relationship to the material and normative pressures that they face.

It is within the gay studies tradition that this article makes its intervention into chemsex research. In line with Race and Hakim, it applies a networked approach to circumvent both the reflexive problematisation of normative subjectivity and its containment to an imagined problem public of "bad gays". To achieve this, the article decentres such publics and instead focuses on chemsex as it operates in porn. Moreover, it applies an affective, networked analysis to understand how desire, 
risk, and sociability are negotiated in chemsex encounters between a range of people, things, and sites that have not been addressed in the literature before.

To understand chemsex, I posit that we must trace how it configures fear and desire into intimate publics. To do so in a digital context, I take into account both content and platform, material expression and pathway/assemblage, human and nonhuman action, and how these aspects come together in collaborative or contentious ways. In other words, this article asks what computational, aesthetic, and communicative processes give shape to a chemsex imaginary on Pornhub, and how dominant and counterpublic scripts are implicated in these. Concretely, the article constructs chemsex publics by assessing video aesthetics, the computational or algorithmic interventions that make such aesthetics recognisable, as well as the audience commenting practices that are presented alongside the videos.

The organisation of the article is as follows: First, I identify and critique common problematisations in chemsex literature, and situate my contribution within a networked and culturally attuned strand of chemsex research. Then, drawing on Berlant (1998) I theorise how fear and pleasure are configured in dominant publics and counterpublics (Warner, 2002). In order to sensitise the analysis to the digital socio-materiality of Pornhub, I draw in studies on platformed porn (Paasonen, 2011; 2018), algorithmic culture (Bucher, 2018), and social media user production (Burgess, 2006). Subsequently, I present my digital ethnographic approach to generating data, as well as some key aspects of the dataset, including how it is coded, processed and stored. I then move on to analyse the archive, arguing that the archive is loosely organised around two principles: implied and explicit drug use. After detailing how algorithms and users participate in this distinction, I move on to the final section, suggesting that there are two separate publics at play: one that cautiously engages with the erotics of drugged disinhibition, and a counterpublic that takes its erotic charge from the hegemonic marking of drugged sex as "transgressive". In conclusion, I argue for a networked approach to the study of chemsex as an affective reality that spans many (digital) sites and more publics than those typically imagined in such research.

\section{Chemsex as an object of fear and desire}

The particularly influential documentary "Chemsex" (Fairman and Gogarty, 2015) represents chemsex as a disconcerting phenomenon that, Race argues, is moralising and in effect works to "...marginalis[e] the vast majority of occasional users" (Race, 2015). Clearly, chemsex is an object that attracts fearful representations and responses. With drug use operating as an agent of chaos in the public imagination, not least those drugs being delivered intravenously, fear is arguably deeply enmeshed with how the practice of chemsex is imagined. However, rather than arguing for the study of chemsex without or somehow beyond fear, I contend that a critical investigation should reflect on the ways in which the erotic thrust of chemsex might weave together fear with desire, and what the role of drug use is in such assemblage.

While chemsex is an object of fear, this does not mean that other, more normative, figures and practices are not involved in fear. In fact, by extending Berlant's analysis of intimacy (1998), 
fearfulness can be understood as the affective thrust that in effect works to separate hegemonic intimate publics from nonnormative ones. In other words, normative erotics, whatever they may be in a given time and place, can emerge only because exclusion through negative emotions separate out the non-normative. Based on this affective, theoretical framework, we can speculate about the power of fear even when it is not readily registered upon the face or the body, but only emerges as an action, an orientation away from. Such a speculative approach is needed to study the absent presence of fear, which in turn is a necessary intervention to explore the ways that hegemonic value systems create normative publics by marking certain objects as outside, as something to fear.

To interrogate how fear and desire are configured in chemsex on Pornhub, I turn to Warner's concept of "publics" (2002). Rather than imagining discourse as pertaining to well-defined groups of people with their own stable sets of traits and values, a public describes something that "comes into being only in relation to texts and their circulation" (ibid.: 413). This approach frees the analysis from a search for the "typical" chemsex genre that so easily reproduces the idea of the problematic chemsex user. While 'publics' do not express the social totality that the notion of 'The Public' carries, they may still be sites of "world-making”. What Warner calls 'counterpublics' are in fact are constituted "...through a conflictual relation to the dominant public" (ibid.: 423) which is manifested in "speech genres" and "modes of address" (ibid.: 424). From this perspective, and with Race's and Hakim's works in mind, the desires and pleasures of chemsex cannot be represented in publics that align themselves with The Public, without fear or other negative emotions blocking access to such pleasures. Conversely, chemsex counterpublics set out to eroticise drug use (including representations of drug use) in ways that are indebted to their exclusion from The Public; the fear that blocks pleasure in normative publics is made to heighten pleasure in chemsex counterpublics. In other words, chemsex may be sexy because it is marked as "transgressive" and its placement outside the 'charmed circle' of normative sexuality (Rubin, 1994). Analysis of publics should trace normative struggle in discourse-affective "speech" acts but sidestep work that originates them within specific subcultural groups. In the context of Pornhub, this approach allows us to trace speech acts of both humans and non-humans. There may be competing publics, and they may configure desire and fear according to dominant or counterpublic scripts.

Warner's publics only tangentially consider what the material reality of the internet does to the operation of publics, and so to gain a firmer grasp of chemsex publics on Pornhub, I turn to more recent works on porn, platform, and genre studies.

\section{Algorithmic and user organisation and production}

In Carnal Resonance (2011) Susanna Paasonen argues for the study of platformed porn through the lens of affect, how it moves, materialises and transforms across several material infrastructures:

the materiality of the bodies performing in and viewing pornography; the technological objects, protocols, networks, and platforms through which porn materializes as certain kinds of objects; the materiality of perception; and the textures of pornographic images (ibid.: 8) 
From this perspective, we can think of fear and desire as configured at and across such sites, requiring that the researcher assembles chemsex in a series of cuts that draw together certain aspects, leaving others behind. These cuts mirror how online porn is not merely there, in stable digital archives, but rather emerges as countless videos are being organised into more or less stable archives through sorting work done by human and nonhuman actors alike. This paper focuses on the sociotechnical flows between content, users, and technology. Concretely, I conceptualise these dimensions as the genre aesthetics of the video content, the user discourse and sociability as it appears in the video comments, as well as the algorithmic interventions that Pornhub's search functionality and suggestions engine offers. I will weave together these dimensions as it is in their interplay that chemsex publics emerge.

While the analysis of genre and user discourse are well-established modes of inquiry, the cultural study of algorithms is a newer and less unified analytical strand. In the literature, algorithms are typically interrogated as cultural agents within a culture, and as cultures in and of themselves (Beer, 2017; Seaver, 2017; Bucher, 2012). Bucher usefully suggests a 'technicity of attention' operates through anticipation (Bucher, 2012: 13-14). For example, Pornhub's interface serves up suggestions of content and search terms based on your previous input. Thinking further about what this process does, I find it useful to turn to the concept of 'vernacular creativity' (Burgess, 2006) that by and large conceptualises such anticipatory production from the side of the social media user. It recognises that social media affordances create fertile grounds for the combination and recombination of familiar material and immaterial cultural resources into novel expressions (ibid.: 206). By applying this logic to both algorithmic and user interventions and production in social media, we can trace how humans and nonhumans collaborate or clash in the organisation of material and imaginaries. They can be more or less aligned and cooperative or, conversely, actively work on and against each other, creating a field in which users and algorithms struggle to create meaning.

Overall, my study of chemsex on Pornhub maps how users, algorithms and the researcher are all involved in the formulation of chemsex publics. Concretely, I look at search activity, suggested search terms and videos, the genre characteristics of video clips, and the discourse found in the comments section. To produce relevant material, I apply an ethnographic approach which is described in the following section.

\section{Methodology and material}

A purpose of this study is to uncover how visual genres and modes of engaging emerge as Pornhub users engage with algorithmic possibilities, and conversely when Pornhub's algorithms engage with its knowledge of the user and their actions. The user and the algorithm are brought into being in what Annemarie Mol calls 'praxiography': multiple materialities that emerge in practical engagements (Mol 2002: 82). To produce such a rich data archive, I have deployed the walkthrough method (Light et al., 2018). This method enables a detailed description of the process of navigating the Pornhub site, and how I arrive at the videos and comments included in the sample. I have chosen to focus on what kinds of chemsex publics emerge when Pornhub is accessed from a 
logged-in user profile. In creating the profile, I marked myself as interested in men having sex with men only, which speculatively may produce algorithmic and content engagements that somewhat align with this interest. So, while this study expands the study of chemsex, it does so from a sociomaterial starting point of users interested in gay porn content.

The walkthrough details how the Pornhub algorithms seem to dynamically respond to my inquiries and lead me to explore sometimes unexpected pathways. Algorithmic interventions included are search suggestions, related search terms, and related videos sections, as well as (partly) related playlists. These playlists, however, constitute a liminal category in that while they are offered in context through algorithmic prediction, they also express user-produced creativity since they are assembled, categorised and maintained by Pornhub prosumers.

The videos have been selected from four points of entry, namely two keyword searches and two user-generated playlists (Table 1). The keyword searches are "chemsex" and "chemsex slam", the latter emerging as a suggested search term second to "chemsex" (See Figure 1). "Slam" means administering crystal meth intravenously. The lack of videos of smoking crystal meth found through the search in the sample reflects the algorithmic attention which turns the user turn towards injection. Videos of people smoking crystal meth $(n=11)$ were, however, included in the sample by way of the two playlists "ParTy" $(n=13)$ and "PnPartyMethysexxxMixxx" $(n=10)$ that emerged as related content in the two searches. Videos found through the search and playlists were chosen as they appeared on-screen; for those searched this was the "most relevant videos"; for playlists it is unclear how the videos are organised.

\begin{tabular}{|l|r|}
\hline Discovery method & Video count \\
\hline Search “chemsex" & 11 \\
\hline Search “chemsex slam” & 7 \\
\hline Playlist "ParTy" & 13 \\
\hline $\begin{array}{l}\text { Playlist } \\
\text { "PnPartyMethysexxxMixxx" }\end{array}$ & 10 \\
\hline Total & $\mathbf{4 1}$ \\
\hline
\end{tabular}

Table 1: Video sample $(n=41)$.

Overall, the data archive consists of a walkthrough diary, 41 Pornhub videos, and in total 450 comments made in response to the videos at the time of harvest. General video data has been collected, including title, runtime, and number of views. The videos were subjected to thematic coding of basic differences in format, types of physical encounters, and types of drug encounters, including how explicitly the latter were aesthetically represented. The material was loaded into a qualitative data analysis program and subjected to four rounds of coding. Initial rounds explored the 
data through abductive coding for emerging themes, and later coding rounds included deductive automatic coding via keyword searches. The major thematic code groups were vernacular creativity, normativity, affect, drugs, sociability, HIV, sex, and video origin.

\begin{tabular}{|l|l|}
\hline chemsex & Q \\
\hline Video Results \\
\hline Chemsex \\
Slam Chemsex \\
Bareback Chemsex \\
Chemsex Asian \\
\hline
\end{tabular}

Figure 1: Suggested searches when searching for "chemsex".

A specially developed script in the programming language $\mathrm{R}$ was used to harvest Pornhub usernames and comments from the 41 videos. ${ }^{2}$ The general evaluation of risk, harm, and value draws on the ethical framework used in much internet research (Markham and Buchanan, 2012). Storing usernames elevates the risks of identification, which in the context of such marginalised and taboo practices may lead to serious social harm. However, because Pornhub usernames are often creative pseudonyms referencing body parts, porn categories, and desires, it was deemed that they might tell important stories about the backgrounds and values of the users. To minimise risk, these pseudonyms were redacted as soon as the analysis was completed. The comments were scrubbed for data contributing to the identification of persons. Key videos have been stored locally, with only hyperlinks saved for the rest. This has meant the exclusion of two videos from the original sample as their deletion meant that their initial categorisation could not be verified.

Notably, there is no content overlap between the four points of entry, which indicates that the amount and diversification in what may count as chemsex porn on Pornhub is so big that algorithmic and user-produced attentions often create very distinct archives. In other words, the material reflects that experiences with chemsex on Pornhub is highly contingent and may produce unique user experiences time after time. This strengthens the reliability of the claim that the production and consumption of chemsex on Pornhub mostly depends on which organisational mechanism of Pornhub is being used to find such content. Further, it should be noted that while definitions of chemsex in the literature mostly include a range of drugs, the data material indicates that the types of attention produced on Pornhub typically centres on crystal meth. The reasons and implications for this are beyond the scope of this article.

\section{Archives of implied and explicit drug use}

To find and consume chemsex material on Pornhub, as is the case for finding anything online, its users must have some previous knowledge of the vernacular, for them to be able to search for it. The deeper the knowledge, the better the search results, with more videos of desired content, and 
less "false positives". Like YouTube and other DIY content platforms, refined possibilities for exploration is key to Pornhub's success, creating never-ending chains of content recommendations and consumption ("rabbit holes" in everyday internet vernacular). So, while the aesthetic properties of the videos and the discourse in the comments section are key elements to chemsex publics, so is the organisational work carried out, in part, by algorithms trained by the tastes of the community's activity. Consider this encounter with Pornhub's search interface:

I enter Pornhub from "incognito mode" in the browser as I assume many users access it. I have created an account and I'm browsing from it to see what Pornhub feeds back to me. First off I search for chemsex, and before I hit enter I am presented with a dropdown menu of what seems to be elaborations or refinements of my search. They are "Chemsex", "Slam chemsex", "Bareback chemsex", and "Chemsex Asian". (from walkthrough diary)

The chemsex search is presented in Figure 1, with further algorithmic interventions in Figure 2 and Figure 3. In the case of the "chemsex" search, the user is presented with algorithmic intervention that expands chemsex vernacular to short forms for crystal meth (meth, tina), the chemsex event (meth fuck, drug sex, pnp) and drug consumption practice (slam).

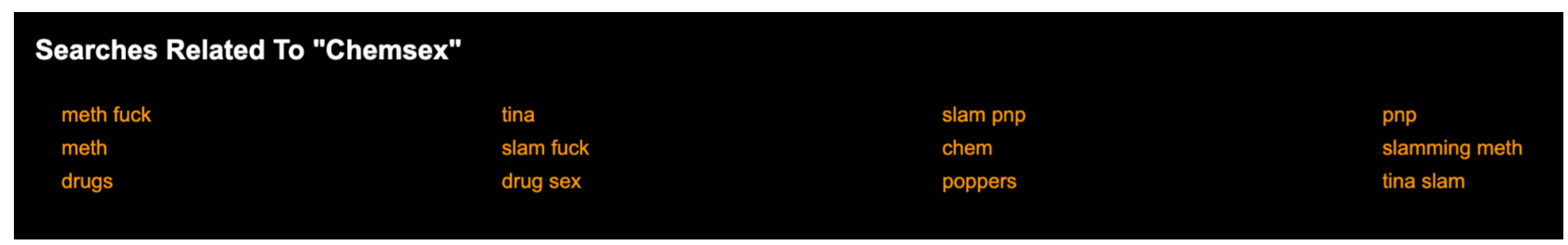

Figure 2: Suggested search terms after searching for "chemsex"

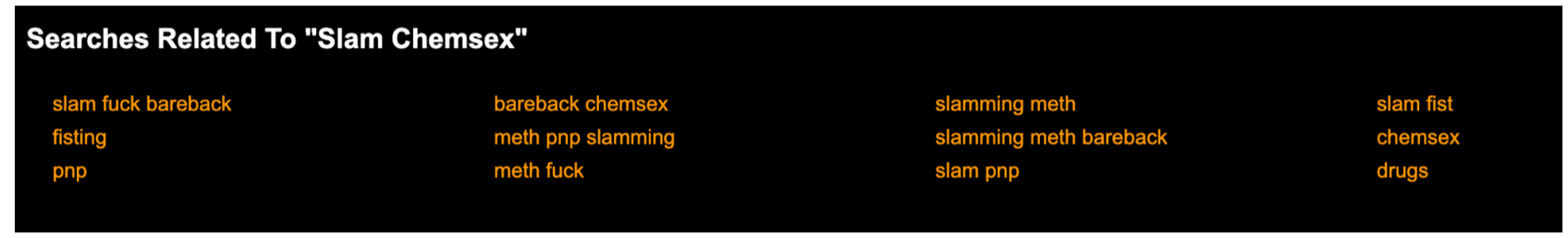

Figure 3: Suggested search terms after searching for "slam chemsex"

Generally, by being presented with other terms, the user's potential access to online chemsex is both widened and conceptually deepened or refined. Conversely, the search suggestions include "bareback" and "Asian", which do not exclusively pertain to chemsex but perform as separate, popular porn genres on their own. Thus, this algorithmic intervention invites the user to explore the inner workings of a chemsex public, as well as its networked connections to, and overlap with, other erotic publics. While the aesthetic and vernacular creativity that defines chemsex emerges among enthusiasts who actively produce, distribute and comment on videos, Pornhub's algorithmic intervention enables such knowledge to spread to more casual users with little somatic, embodied chemsex experience themselves.

The following diary excerpt exploring the "chemsex slam" search suggestion illustrates how a user's experience with chemsex publics can widen, deepen, and multiply meaning making: 
I click on 'Chemsex' and is presented with "most relevant video results". It shows 20 out of 131 videos. That's not a lot of videos, giving me the impression that chemsex is only sparsely part of the erotics on Pornhub. But when I navigate back and choose the "chemsex slam" search option, 1140 videos appear. With the added word "slam" a range of other videos are shown. The search seems to introduce two types of videos that align with two vernacular meanings of the word "slam": 1) using crystal meth by injection and perhaps having sex on this particular kind of high, or 2) being fucked hard. (from walkthrough diary)

The algorithmically produced related searches constitute an intervention that does vernacular and aesthetic work, teaching the user how to properly consume chemsex online, while also introducing semantic noise. In this instance, the algorithmically surfaced vernacular expands the chemsex archive tenfold, a dramatic increase that changes the first experience of chemsex as a marginal, almost non-existent phenomenon on Pornhub, to one with a significant amount of content available. The prominent placement of the slam vernacular, along with its combination with chemsex, indicates to the user that might have never heard of it before, that it is a) related to chemsex, and b) a significant part of chemsex. Clicking through presents the user with video content that might teach them about chemsex's cultural meaning. With all things equal, the precision with which the user can understand the concept increases with the number of data points available (videos, tags, titles, comments), while also depending on the algorithm's ability to produce relevant and coherent sets of data points. The double meaning of "slam" offers a great example of how the algorithm might reproduce and enhance semantic noise, requiring a fair bit of work by the user to parse. Such decoding might of course not be undertaken at all, creating a chemsex experience that has as much to do with hard intercourse as it has with drug-taking.

\begin{tabular}{|l|r|r|}
\hline Videos & Implied drug use & Explicit drug use \\
\hline Amount (n) & 18 & 23 \\
\hline Subject to commenting (\%) & 23 & 58 \\
\hline Median duration (minutes) & 8 & 3 \\
\hline Average duration (minutes) & 9 & 10 \\
\hline Found through search & 16 & 2 \\
\hline Found through playlist & 2 & 21 \\
\hline
\end{tabular}

Table 2: Characteristics of videos with either implied or explicit drug use

Overall, I find that the collected videos can be separated into two categories (see Table 2). Those that only imply drug use, and those that show it explicitly. The relevance of this distinction is strengthened by the fact that the videos also differ when it comes to duration, how they were found, and if they are commented upon by other users. These archives form the basis for the distinctions made in the next two sections. 


\section{Cautious erotics of disinhibition}

In some videos, beyond the search term, no chemsex is implied in any textual elements visible in the user interface. While such videos might be treated as outliers, or simply as non-representative of what chemsex is, I would argue that because such "faulty" inclusion is typical for online search activity, they should not be eliminated from the analysis. Rather, because the chemsex-searching user finds them, they have the potential to be part of what is imagined and consumed as chemsex, even though they display no textual properties recognisable as such. An example of this is the video "Blushed young twinky slammed by his big dicked lover". While displaying no direct drug use, it has probably been included in the search results because of the word "slammed" in the title, in this case not referring to administering crystal meth intravenously but being fucked very hard. This linguistic unclarity exemplifies how algorithmic exploration of chemsex produces results in more or less transparent ways to include videos that are semiotically unrecognisable as chemsex.

Other non-chemsex videos in the sample feature partly Mandarin titles such as “粗過六被十九歲帛 長十九幹了! A TWINK FUCKING ME BY HIS 19CM!". While the Mandarin text does not refer directly to chemsex, Chinese users, as well as Pornhub's search algorithm, might be able to recognise Mandarin chemsex vernacular that I, as someone who does not know the language and its particular linguistic codes, am not. This brings to our attention that while the vernacular creativity around chemsex by and large operates and is understood transnationally, local variations might develop.

In other videos, the performers seen in the videos are implied to be under the influence of drugs (Figure 4). These videos show men having penetrative sex, sometimes in groups. They adhere to the typical DIY porn format and aesthetic of non-storied, non-professional looking performers, in nonstudio setups and with little postproduction such as editing and colour grading. Their bodily interactions are centred, and no drugs or their delivery systems are ever visible in the frame. However, the extra-textual video titles heavily imply drug use. Examples include "Slammed daddy and boys chemsex sweat fuck", "ParTied up T wink Fuck" and "raw chemfun". Thus, the owners of the videos invite them to be read as chemsex encounters. 


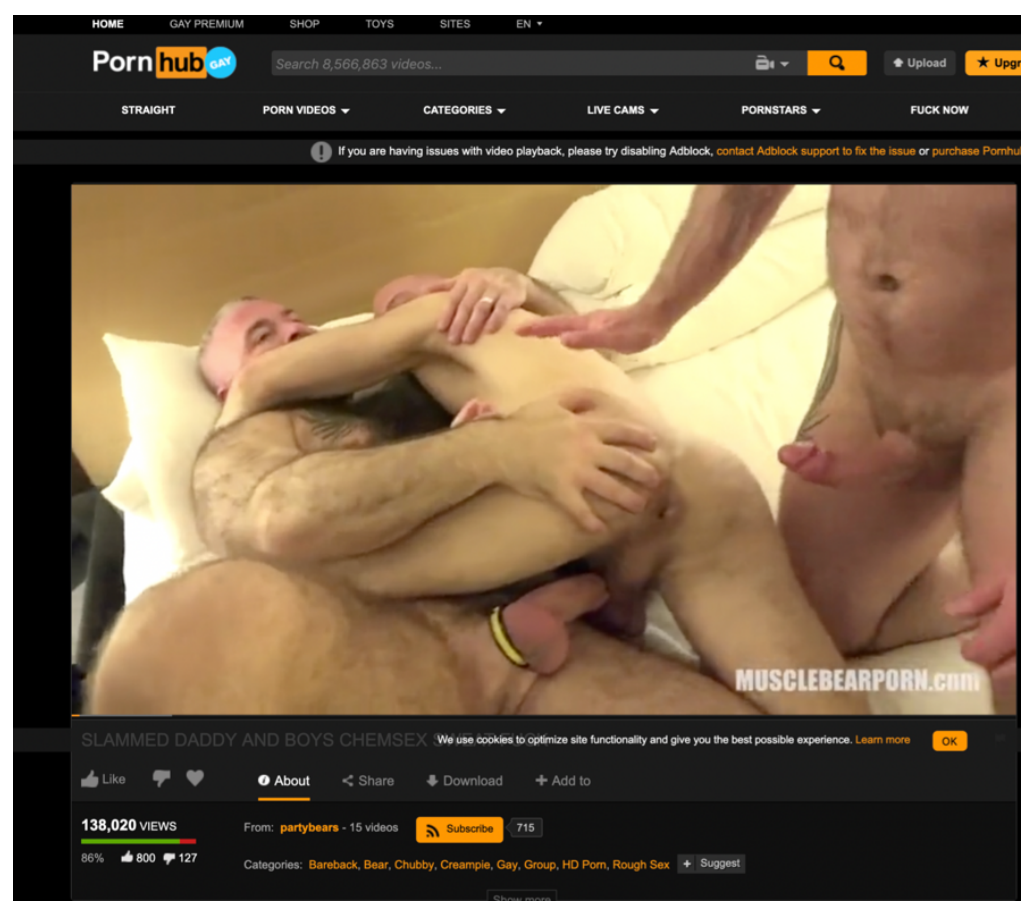

Figure 4: Example of chemsex being implied in the video description

All the videos emerge from searches for chemsex, not from chemsex curated playlists. That means that searching for chemsex content compiles an archive of videos that, to a higher degree than playlist curated videos, exclusively use extra-textual signifiers to frame the aesthetic performance as a chemsex encounter. In fact, videos with no or implied drug use make up the vast majority of the videos produced from searching. Thus, the search experience is one that engages with drugged disinhibition, in a way that brackets out the act of drug consumption itself. While this archive is not assembled by a human or nonhuman entity according to a singular vision, it nevertheless emerges as a public with distinctive traits. It seems to operate, in part, along dominant sexual scripts of drug use being incompatible with "pure" and "real" sexuality publics. It has a cautious quality to it, turning away from drugged pleasure that falls outside the charmed circle (Rubin, 1994), but still remaining somewhat available to its effects.

Such caution can also be seen in the vagueness of the comments to these videos. The overwhelming majority of comments across all genres and origins, but especially for those belong to this public, are emotive, specifically expressing positive emotions. Most comments are short, with various creative formatting choices reproducing the emotive response. One example is the reliance on and remixing of the rather broad word "hot":

hot

Yes please! so hot... 


\section{So Fucking Hot!}

soooo fuckin hot!

The short form and limited vocabulary evokes the notion of affect as something prediscursive (Massumi, 2002), while the experimental formatting brings to mind how affect in such vocabulary constitutes a flow that animates materiality. The reality of drug-taking remains unrecognised while its affective potentialities are discursively consumed. In continuation of this, comments to videos belonging to the cautious erotic public engage more with other aspects of the depicted encounter. Examples are:

love the bottom calling him daddy 3

OMG so hot!. Love the way they fuck...tender...lusty, powerful and careful at the same time...Oh, i wish i could have those bears with me to fuck me like that!!!!

I want to be on the top and fuck a bottom just like that

Here, the erotics of chemsex disinhibition is consumed much like other DIY porn videos, thus ignoring the work that metadata such as the video title does to frame it as chemsex. The video performers display an interest in chemsex that to a large extent is not honoured or replicated in the audience. It remains unclear to which degree this reflects the commenters' unawareness of, or disinterest in, this aspect, or if they are cautiously circumventing dominant scripts in order to access chemsex pleasures.

Overall though, the public that the computational interventions of the search algorithm have brought into discursive existence is one that aligns with hegemonic scripts of sexual intimacy. However, by representing its effects of disinhibition alone, the public forms around a cautious mode of engaging with pleasure that threatens. A mode that, we may speculate, remains somewhat accessible to people whose sexual orientation operates through such hegemonic scripts. Conversely, audiences with personal, somatic chemsex experience might also simply silently consume these videos, drawing other forms of pleasure from it. However, for those more intently interested in and comfortable with chemsex aesthetics, user-generated playlists offer a much denser and explicit experience, as well as a more engaged discursive community. The next section turns to this.

\section{Counterpublic erotics of transgression}

Another, more clear-cut, chemsex public emerges in the data. These videos display sexual acts as well as explicit drug use not only in the extra-text and meta-text but in the videos themselves. Drug administration by injection or smoking is shown, with some of the videos also showing its effect on the bodies followed by an erotic encounter. The viewer gets visual proof of drug-taking which invites the reading of the sexual encounter as chemically enhanced and disinhibited. The videos are 
by and large found through user-curated chemsex playlists, not from search terms. This, combined with the fact that implied chemsex videos take up most space in searches, reinforces the distinction between the cautious chemsex public that reveals itself when scrolling the search results, and the more explicit eroticisation of drug consumption in playlists.

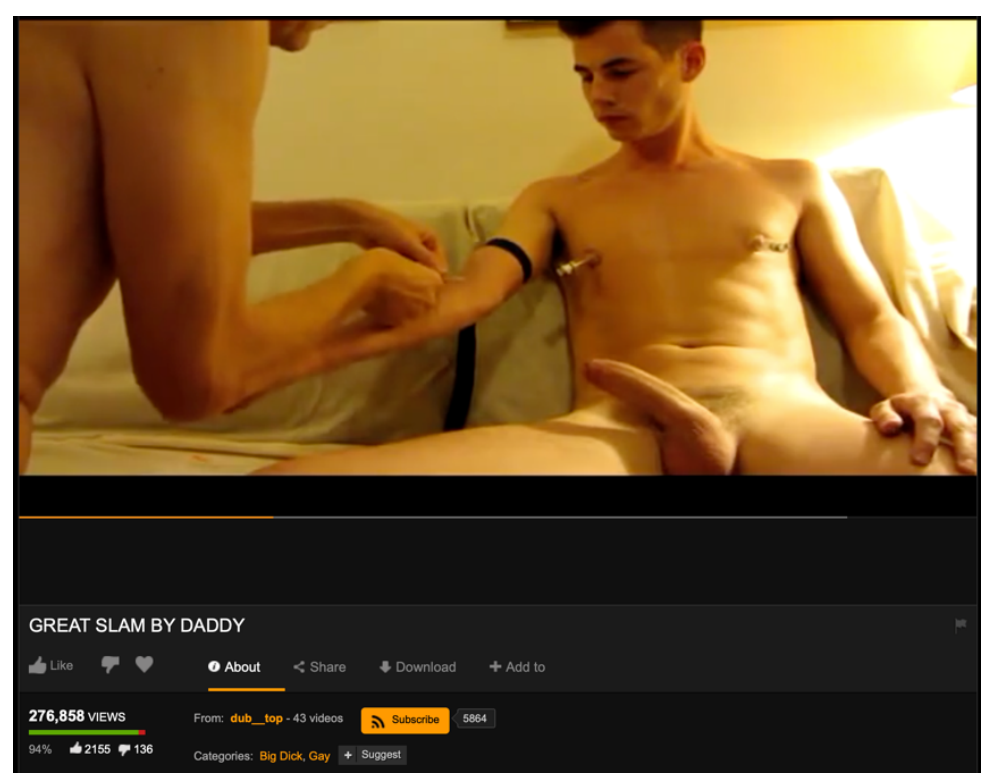

Figure 5: Example of video in which crystal meth is injected ("slammed")

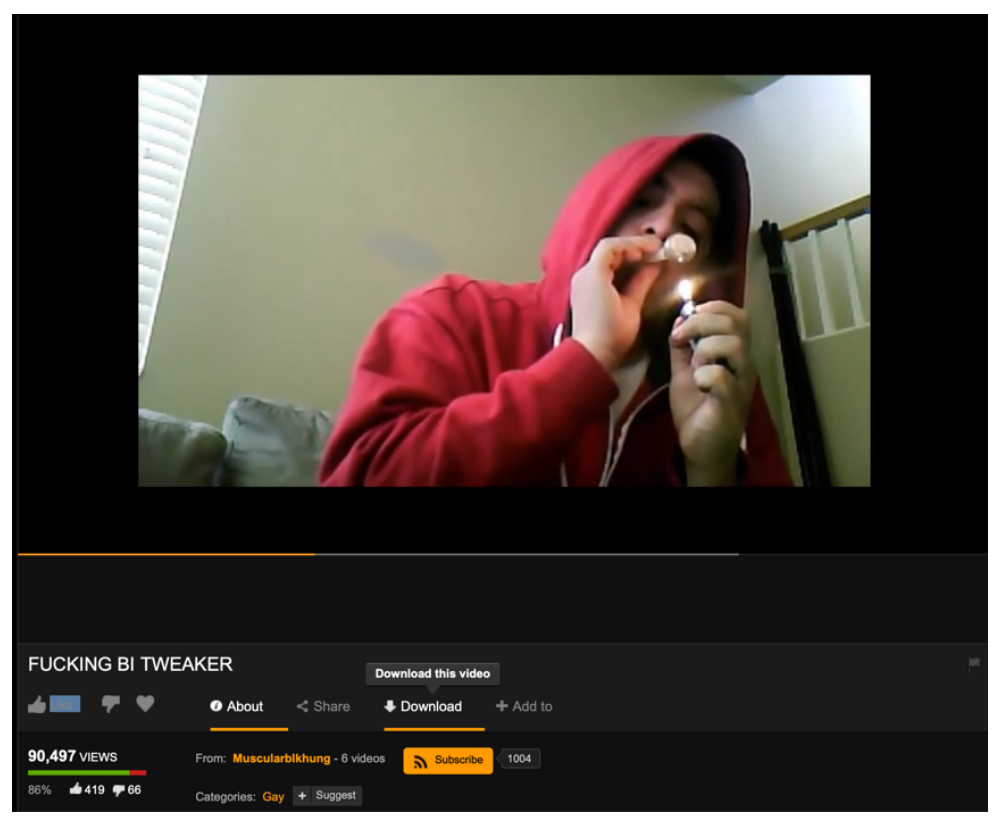

Figure 6: Example of video in which crystal meth is smoked ("clouds")

Some videos centre on the intravenous administration of crystal meth (Figure 5), and more or less exclude sexual activity such as kissing, masturbation or anal penetration. While these have much in common with the previously described videos showing drug use and its sexual effects, this subset is 
distinct in a couple of ways. First of all, they are the shortest of all the videos, with a typical runtime of two minutes, showing little variation. If we take their aesthetic as inherently erotic, then it is reasonable to conclude that the injection of the drug constitutes the erotic moment, that drug-taking provides the erotic thrust. In other words, these videos' erotics have less to do with penetrative, gay porn, and more to do with the eroticisation of drug-taking in itself. This aligns with another study (Race et al., forthcoming) that finds that the administration of crystal meth in 'slamsex' operates similarly to a sexual preference. In that sense, in the same vein as the non-chemsex videos, the aesthetic represents a liminal chemsex public that does operate in an erotic register, but does so without the traits of the typical sexual encounter in porn. Notably, unlike injecting, the act of smoking crystal meth, while well-represented in the sample (for a visual example, see Figure 6), is not fetishized to the degree that it performs as the only erotic moment in a video.

Regardless, these videos and their expert organisation into playlists do not shy away from drug use; in fact, in the context of dominant sexual scripts, their eradication of fearfulness and shame can be seen as counterpublic resistance. Within them sexual tension is not blocked by fear; on the contrary, the marking of drug use as "transgressive" heightens the pleasure that can be taken from them. This is also reflected in the comments section. In contrast to commenting cultures among videos with implicit drug use, these video comments perform an audience for whom sexual drug use is clearly registered, personally related to, and taken pleasure in. Examples include:

Love to blow some clouds with ya!!

want to smoke together wit you... [video conferencing service 1] or [video conferencing service 2$] ?^{3}$

the hoTTesT man on here! i love watching you hit the pipe and suck!!!

hoT fucking parTy, love hitting the pipe blow cock

love to smoke then play with dicks

The audience emerges as well-versed in both online and offline chemsex culture and practice. Use of vernacular like "clouds" and the uppercase Ts demonstrate someone comfortable with the codes of the culture, and knowledge about delivery systems such as pipes reveal drug experience that goes beyond Pornhub. Furthermore, the occasional reference to other mediated forms of chemsex sexual sociability, such as using video conferencing services (Møller, 2020), calls attention to the fact that to some, Pornhub is only one media site in their digital chemsex ensemble or repertoire (Hasebrink and Hepp, 2017).

In some instances though, large and refined vocabularies are used along with experimental formatting to produce vivid erotic performances: 
HOT, HOT vid!!! 1st, jusT the premise, \& horny, BUTCH, methed-up head seeking some ALL niTe cock-WORSHIP \& that PitBull hanging around.... The fuk'r has a GREAT, flaccid cock \& NO probs getting FULLY aroused while clouding up, HUGE Turn-on!!!!!! Then, he has that HOT PitBull wandering around, calmly, as IF he's Trained to be quieT \& jusT observe, buT...ThaT's jusT how the 2 begin their carefully rehersed, well-orchesTraTed seducTion.... As the sTudly thug geTs me nice \& high, smoking on his pipe (user comment to video)

The comment praises the video and the performers for their aesthetics, for their technical achievements, and for how they manage to arouse the user. The uppercase Ts denote 'tina' or crystal meth, thus succinctly describing a "drugged hotness". In other words, the comment performs the sexual appreciation of both chemsex intra-action (Barad, 1996) and interaction. Intra-action in that it performs and appreciates the drugs' modulating effects on the senses by modulating words through capitalisation of Ts, thus quite literally representing the fundamental chemical alteration of experience, and interaction because the comment appreciates the sensations that drug-using bodies are able to produce in other bodies. It is a discursive strategy that performs the erotic arousal of the commenter, while placing them in a sort of affective, if not situational, proximity to the video performers.

Only a handful of comments express negative emotions or evaluation, with the overall experience being that of positivity and erotic play. Such play is mostly performative and only dependent on the other users as a passive audience; most comments do not invite direct responses, which supports the reading of them as affective responses and erotic performances more than forms of sociability. However, a small subset of comments do register as sociable. Mostly social activity emerges when the video owner actively responds to comments or questions. Often, when the owner is perceived to be a performer in the video this leads to more or less serious planning of meetups inspired by the encounters depicted in the videos:

user: $\mathrm{i}$ want $\mathrm{u}$ blowing clouds on my cock as u deep throat my meat owner: Would LOVE too.

user : I want to come over and party

owner: Come on Stud! I'll party with you anytime

user: [HOTTT]

owner: I'm glad you liked it.

Here, an intimate charge seems to be enacted among the video owner and user, drawing them together into a potential erotic encounter, which in turn produces intimacy effects among the wider audience that is witnessing the interaction.

Overall, the explicit erotics of drug use emerge from communities of highly engaged Pornhub users that assemble playlists with direct representations of not only drugged bodies, but the act of consuming drugs. Whether or not the typical audience member has any somatic experience 
resembling what is seen on-screen is beyond the scope of this article. However, as a discursive community, the audience acts as a counterpublic in the sense that it is organised around openly taking pleasure in an object that is marked as something to be feared.

\section{Conclusion}

In contemporary society, hegemonic scripts of sexual intimacy make sexualised drug use a transgressive practice placed outside the charmed circle. This placement works by making drug use an object of fear that blocks the ability to take pleasure in its effects. It is in this light that we must understand and critique the narrow focus on "problematic" behaviour that most academic and news discourse on chemsex concerns itself with. By studying drug use on Pornhub this article circumvents such overdetermined discourse by leveraging porn as a resource for rich and contradictory expressions of erotic repertoires. The article finds that Pornhub indeed serves as a space for experimentation with drugged sexuality that to varying degrees breaks with hegemonic scripts of sexual intimacy.

As a cultural study of chemsex, the study finds that online chemsex operates as a wide range of aesthetic and discursive variations, in some cases within which drug use is only tangentially present. Pornhub's video archives are, to varying degrees, assembled by humans or non-humans and they serve as interventions that generate different visions of chemsex. This happens in intraactive processes of algorithms-user organisational flows that territorialise two configurations of fear and desire. The analysis has shown that such flows look very different depending on whether they are mostly machine driven or user driven. Algorithms typically produce publics that either do not read as chemsex, or that cautiously sidestep dominant scripts to access pleasures of drugged disinhibition, while users typically surface chemsex counterpublics of transgressive pleasure.

On the one hand, videos aggregated through the search produce less drug-explicit content, while being more aesthetically attuned to mainstream DIY porn visual tropes and with user engagement, beyond watching, comprising non-drug related praise. Thus, in search results, chemsex, for the most part, is not present and only alluded to. The assembled videos and discursive communities around them describe what I have called a cautious erotics of disinhibition. Knowing the lives and motivations of audiences are beyond the scope of this article. That being said, the videos that focus on the disinhibitive effects on the body without directly showing drug consumption, could be seen as producing a public that, while still oriented towards a transgressive subject, is relatively more respectable and aligned with homonormative politics of assimilation.

On the other hand, the ability to produce and share playlists, and have them algorithmically promoted to relevant audiences, supports the formation of an erotic counterpublic that explicitly consumes the administering of drugs. These videos are also subject to higher levels of socialisation that appreciate drug use and culture. In other words, this public draws its erotic charge from the consumption of crystal meth, in a way that not merely disregards it place outside the normative space of pleasure but in counterpublic fashion is in fact motivated by such placement. 
We may think about these differences through which erotic publics are activated as representing variations in audiences, with different personal biographies or, to take it further, different experiences with taking drugs (while having sex), with drugged disinhibition being subject to very different fear-desire configurations.

Taking a step back, I would argue that the basic fact that chemsex aesthetics circulate in contemporary platformed gay porn indicates that chemsex is implicated in a much wider array of gay sexual orientations than the focus on the somatic reality of private sex parties has so far revealed. While chemsex may happen in physical meetings, it is more than that: it is an intra-active event in which drugged sexual affects along with normativities flow though emerging networks of bodies, sites, and technologies. By insisting that chemsex is also constituted by algorithms, content producers, and user audiences, the containment of a problematic public becomes more difficult, instead rendering us sensitive to the fact that chemsex porn contains a multitude of erotic orientations that cut across more or less normative publics. As such, chemsex is better conceived of as an erotic register consumed in very different ways, including by those who live lives not currently recognisable as entangled with chemsex. Further interview-driven studies are needed to understand the variations in chemsex experience, including how media take part in such experience and practice.

\section{References}

Barad K (1996) Meeting the Universe Halfway: Realism and Social Constructivism without Contradiction. Feminism, Science, and the Philosophy of Science: 161-194. DOI: 10.1007/978-94-009-1742-2_9.

Beer D (2017) The social power of algorithms. Information, Communication \& Society 20(1): 1-13. DOI: 10.1080/1369118X.2016.1216147.

Berlant L (1998) Intimacy: A Special Issue. Critical Inquiry 24(2): 281-286. DOI: 10.1086/448875.

Bourne A, Reid D, Hickson F, et al. (2014) The Chemsex Study: drug use in sexual settings among gay and bisexual men in Lambeth, Southwark and Lewisham. Public Health England. Available at: http://researchonline.lshtm.ac.uk/2197245/ (accessed 4 December 2016).

Bourne A, Reid D, Hickson F, et al. (2015) Illicit drug use in sexual settings ('chemsex') and HIV/STI transmission risk behaviour among gay men in South London: findings from a qualitative study. Sexually transmitted infections 91(8): 564-568. DOI: 10.1136/sextrans2015-052052.

Bucher T (2012) A technicity of attention: How software'makes sense'. Culture machine 13: 1-23.

Bucher T (2018) If...Then: Algorithmic Power and Politics. New York: Oxford University Press.

Burgess J (2006) Hearing ordinary voices: Cultural studies, vernacular creativity and digital storytelling. Continuum 20(2): 201-214. 
Dean T (2009) Unlimited Intimacy: Reflections on the Subculture of Barebacking. Chicago \& London: The University of Chicago Press.

Fairman W and Gogarty M (2015) Chemsex. UK: Vice. Available at: http://www.imdb.com/title/tt5073738/ (accessed 2 October 2020).

Hasebrink U and Hepp A (2017) How to research cross-media practices? Investigating media repertoires and media ensembles. Convergence 23(4): 362-377.

Light B, Burgess J and Duguay S (2018) The walkthrough method: An approach to the study of apps. New Media \& Society 20(3): 881-900. DOI: 10.1177/1461444816675438.

Markham A and Buchanan E (2012) Ethical Decision-Making and Internet Research Recommendations from the AoIR Ethics Working Committee. Available at: https://aoir.org/reports/ethics2.pdf (accessed 2 October 2020).

Massumi B (2002) Parables for the Virtual: Movement, Affect, Sensation.

Mol A (2002) The Body Multiple: Ontology in Medical Practice. Durham: Duke University Press.

Møller K (2020) Hanging, blowing, slamming and playing: Erotic control and overflow in a digital chemsex scene. Sexualities: 136346072096410. DOI: 10.1177/1363460720964100.

Paasonen S (2011) Carnal Resonance: Affect and Online Pornography. Cambridge: MIT Press.

Puar JK (2007) Terrorist Assemblages: Homonationalism in Queer Times. Durham: Duke University Press.

Race K (2015) Chemsex review: gay sex and drug use demand more careful forms of attention. Available at: http://theconversation.com/chemsex-review-gay-sex-and-drug-use-demandmore-careful-forms-of-attention-51586 (accessed 20 June 2017).

Race K (2018) The Gay Science: Intimate Experiments with the Problem of HIV. London \& New York: Routledge.

Race K, Murphy D, Pienaar K and Lea T (forthcoming) Injecting as a sexual practice: cultural formations of 'slamsex'. Sexualities.

Rubin G (1994) Thinking Sex; notes for a radical theory of the politics of sexuality. In: Abelove H, Barale MA and Halperin DM (eds) The Lesbian and Gay Studies Reader. New York: Routledge: 3-44.

Seaver N (2017) Algorithms as culture: Some tactics for the ethnography of algorithmic systems. Big Data \& Society 4(2): 205395171773810 . DOI: 10.1177/2053951717738104.

Sedgwick EK (1985) Between Men: English Literature and Male Homosocial Desire. Columbia University Press.

Warner M (2002) Publics and counterpublics (abbreviated version). Quarterly Journal of Speech 88(4): 413-425. DOI: 10.1080/00335630209384388. 


\footnotetext{
${ }^{1}$ In a later test run this particular set of related searches has not been replicable, which underscores the challenges of studying the work of algorithms, with their modes of operation for the most part being blackboxed by the companies that own them.

${ }^{2}$ Big thanks to my dear colleague Obaida Hanteer who wrote and executed this script, thus helping create the dataset. ${ }^{3}$ The names of these video conferencing services are redacted to reduce the risk of this publication expediting the rate with which chemsex social scenes that depend on these services are shunned from them.
} 